\title{
$\begin{array}{ll}\text { Research Square } & \text { Preprints are preliminary reports that have not undergone peer review. } \\ \text { They should not be considered conclusive, used to inform clinical practice, }\end{array}$ or referenced by the media as validated information. \\ Quasiparticle Nodal Plane in the Fulde-Ferrell- Larkin-Ovchinnikov state of FeSe
}

Shigeru Kasahara

Kyoto University

Hiroki Suzuki

Kyoto University

Tadashi Machida

RIKEN https://orcid.org/0000-0001-7389-8315

\section{Yuki Sato}

Kyoto University

\section{Yuzuki Ukai}

Kyoto University

Hinako Murayama

Kyoto University

\section{Shota Suetsugu}

Kyoto University https://orcid.org/0000-0003-1597-8784

\section{Yuichi Kasahara}

Kyoto University

\section{Tetsuo Hanaguri}

RIKEN https://orcid.org/0000-0003-2896-0081

\section{Takasada Shibauchi}

University of Tokyo https://orcid.org/0000-0001-5831-4924

Yuji Matsuda ( $\square$ matsuda@scphys.kyoto-u.ac.jp)

Kyoto University https://orcid.org/0000-0001-9947-9418

\section{Article}

Keywords: interacting fermions, Fulde-Ferrell-Larkin-Ovchinnikov (FFLO), Quasiparticle Nodal Plane, FeSe

Posted Date: April 8th, 2021

DOl: https://doi.org/10.21203/rs.3.rs-381469/v1

License: (9) This work is licensed under a Creative Commons Attribution 4.0 International License. 
Version of Record: A version of this preprint was published at Physical Review Letters on December 17th, 2021. See the published version at https://doi.org/10.1103/PhysRevLett.127.257001. 


\title{
Quasiparticle Nodal Plane in the Fulde-Ferrell-Larkin-Ovchinnikov state of FeSe
}

\author{
S. Kasahara ${ }^{1}$, H. Suzuki ${ }^{1}$, T. Machida ${ }^{2}$, Y.Sato ${ }^{1}$, Y. Ukai ${ }^{1}$, H. Murayama ${ }^{1}$, \\ S. Suetsugu ${ }^{1}$, Y. Kasahara ${ }^{1}$, T. Shibauchi ${ }^{3}$, T. Hanaguri ${ }^{2}$, and Y. Matsuda ${ }^{1}$ \\ ${ }^{1}$ Department of Physics, Kyoto University, Kyoto 606-8502, Japan \\ ${ }^{2}$ RIKEN Center for Emergent Matter Science, Wako, Saitama 351-0198, Japan and \\ ${ }^{3}$ Department of Advanced Materials Science, University of Tokyo, Chiba 277-8561, Japan
}

\begin{abstract}
Among exotic pairing states of interacting fermions, the Fulde-Ferrell-Larkin-Ovchinnikov (FFLO) state $[1,2]$, characterized by Cooper pairs condensed at finite momentum, has been a long-sought state that remains unresolved in many classes of systems, including superconductors $[3,4]$ and ultracold atoms $[5]$. A fascinating aspect of the FFLO state is the emergence of periodic nodal planes in real space, but its observation is still lacking. Here we investigate the order parameter structure for $c$-axis fields on a high purity single crystal of FeSe [6]. The heat capacity and magnetic torque provide thermodynamic evidence for a distinct superconducting phase at the low-temperature/high-field corner of the phase diagram [7]. Despite the bulk superconductivity, spectroscopic-imaging scanning tunneling microscopy (SI-STM) performed on the same crystal demonstrates that the superconducting order parameter vanishes at the surface upon entering the high-field phase. These results imply that the planar node induced perpendicular to $H$ is pinned at the surface, providing evidence of the FFLO pairing state with zeroth Landau level.
\end{abstract}

The FFLO state is characterized by the formation of Cooper pairs with nonzero total momentum $(\boldsymbol{k} \uparrow$, $-\boldsymbol{k}+\boldsymbol{q} \downarrow)$, instead of the ordinary Bardeen-CooperSchrieffer (BCS) pairs $(\boldsymbol{k} \uparrow,-\boldsymbol{k} \downarrow)$. The appearance of the $\boldsymbol{q}$-vector leads to spatial symmetry breaking, in addition to $U(1)$-gauge symmetry breaking. As a result, the order parameter $\Delta(\boldsymbol{r})$ of condensed fermion pairs undergoes one-dimensional (1D) spatial modulation, leading to the periodic nodal planes. In superconductors, where the FFLO state appears in a strong external magnetic field, a stringent condition is required for its formation; a sufficiently large Maki parameter, a ratio of the orbital to the Pauli-paramagnetic limiting upper critical fields $\alpha_{M} \equiv \sqrt{2} H_{c 2}^{\text {orb }} / H_{c 2}^{\text {Pauli }} \approx 2\left(m^{*} / m_{e}\right)\left(\Delta_{0} / \varepsilon_{F}\right)>1.5$, is prerequisite, where $m_{e}$ is the free electron mass, $m^{*}$ is the effective mass, $\Delta_{0}$ is the superconducting gap magnitude at zero field and $\varepsilon_{F}$ is the Fermi energy [3]. Such a large Maki parameter is not attained in most superconductors with $\Delta_{0} / \varepsilon_{F} \sim 10^{-3}-10^{-2}$. Promising candidate systems are Josephson coupled layered superconductors in parallel field $[4,8]$ and heavy fermion compounds with large $m^{*}[3,9,10]$. The former includes several quasi-2D organic superconductors and the latter includes $\mathrm{CeCoIn}_{5}$. However, there has been no undisputed experimental verification of the FFLO state, despite some features of the field-induced phase transition in the superconducting state - mainly because direct observation of the planar nodes, which is the most compelling evidence for the FFLO state, has never been reported.

The layered iron-chalcogenide FeSe $\left(T_{c} \approx 9 \mathrm{~K}\right)$ has recently stirred great interest as a promising platform to investigate various exotic superconducting states $[6,11$, 12]. FeSe exhibits a tetragonal to orthorhombic structural transition (nematic transition) at $T_{s} \approx 90 \mathrm{~K}$ without magnetic order [13]. Fermi surface of FeSe consists of a hole pocket at the zone centre and one or two electron pockets at the zone boundary (Figs. 1a and 1b) $[6,14,15]$. A remarkable feature is the emergence of high-field superconducting phase for both field directions parallel $(\boldsymbol{H} \| a b)[16-18]$ and perpendicular $(\boldsymbol{H} \| c)$ to the layer [7]. In particular, for $\boldsymbol{H} \| a b$, a distinct firstorder phase transition deep inside the superconducting phase, which is revealed by a discontinuous jump of the thermal conductivity, and an anomalous enhancement of the upper critical field $H_{c 2}^{a b}$ have been reported [16].

The high-field phases of FeSe have been discussed in
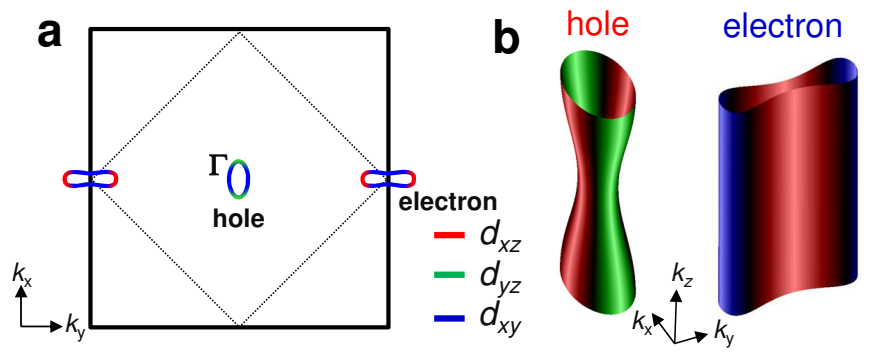

FIG. 1: Schematic illustrations of the Fermi surface of FeSe. a, Schematic figure of the Fermi surface of FeSe in the nematic state in the unfolded Brillouin zone proposed in Ref. [15]. Note that we use the coordinate where the nearestneighbour $\mathrm{Fe}-\mathrm{Fe}$ distance is larger along the $b$ axis ( $y$ direction) than along the $a$ axis ( $x$ direction). Green, red, and blue areas represent the Fermi-surface regions dominated by $d_{y z}, d_{x z}$ and $d_{x y}$ orbitals, respectively. The Fermi energies $\varepsilon_{F}^{h(e)}$ of the hole (electron) pockets are extraordinarily small, $\varepsilon_{F}^{h} \sim 10-15 \mathrm{meV}$ and $\varepsilon_{F}^{e} \sim 5-10 \mathrm{meV}$. b, 3D illustrations of the hole and the electron pockets. The cylindrical hole Fermi surface exhibit a small warping along the $c$ axis. 

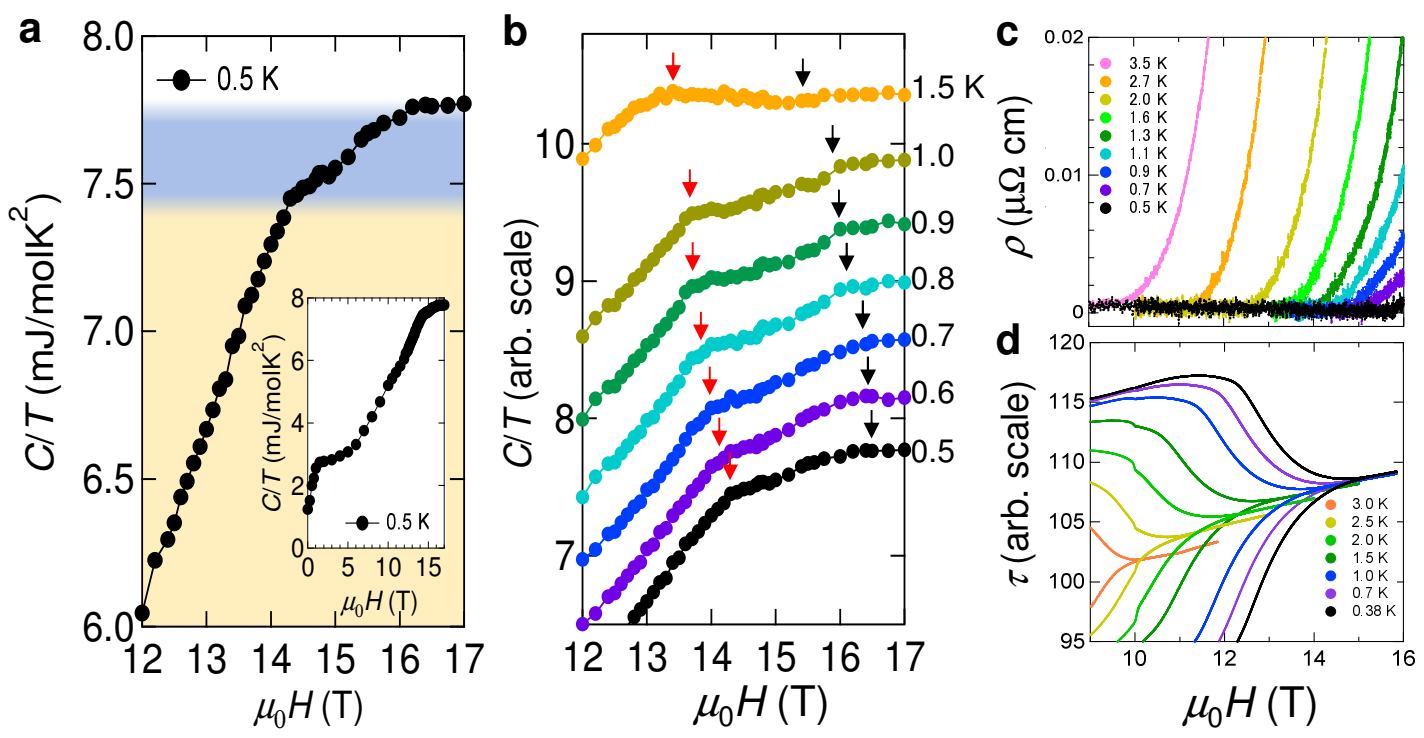

FIG. 2: Magnetic field dependences of heat capacity, resistivity and magnetic torque at low temperatures. a, Magnetic field dependence of the heat capacity, $C(H) / T$, at $0.5 \mathrm{~K}$. The main panel shows $C(H) / T$ at high-fields. The inset shows $C(H) / T$ for the whole magnetic field range from 0 to $17 \mathrm{~T}$. The orange and blue shades denote the two different regimes of the $C(H) / T$ below and above $H^{*}$. b, Magnetic-field dependence of $C(H) / T$ at different temperatures below $T=2.0 \mathrm{~K}$. Each curve is vertically shifted for clarity. The red arrows indicate the kink anomaly at $H^{*}$. The black arrows show the mean field $H_{c 2}^{c}$, above which the $C / T$ becomes field independent. Two kinks are observed up to $1 \mathrm{~K}$. At $1.5 \mathrm{~K}$, although the kinks are not clearly resolved, signatures of the transition at $H^{*}$ and $H_{c 2}^{c}$ can be seen. c, Magnetic-field dependence of the resistivity $\rho$. d, Magnetic-field dependence of the magnetic torque $\tau$. We determined $H_{i r r}$ by the onset field of nonzero resistance, and by closing field of hysteresis loops of magnetic torque.

terms of FFLO state, because of the noticeably enhanced Maki parameter $\left(\alpha_{M} \approx 2.5\right.$ and 5 for hole and electron pocket, respectively) owing to the extremely large ratio of superconducting gap and Fermi energy $\Delta_{0} / \varepsilon_{F}$ and mass enhancement caused by the electron correlation effect $[6$, $7,16]$. The FFLO state is stabilized when a large area of the spin-up Fermi surface is connected to the spindown surface by the $\boldsymbol{q}$-vector. For a $2 \mathrm{D}$ system with a cylindrical Fermi surface, the two Zeeman-split Fermi surfaces touch on a line by a shift of the $\boldsymbol{q}$-vector in the $2 \mathrm{D}$ plane. Thus, in the layered compounds, the FFLO state with $\boldsymbol{H} \| \boldsymbol{q}$ is more favourable in parallel field than in perpendicular field [19]. Therefore, whether the FFLO state is formed for $\boldsymbol{H} \| c$ is a highly non-trivial issue [20, 21].

All measurements were performed on the same crystal (see, Method). This is particularly important because $T_{c}$ and $H_{c 2}$ slightly depend on the samples even from the same batch. To reduce $T_{c}$ distribution caused by the inhomogeneity, we selected a tiny crystal $(\sim 30 \mu \mathrm{g})$ containing extremely small amount of impurities (Figs. S1).

As depicted in the inset of Fig. 2, $C(H) / T$ grows steeply up to $\mu_{0} H \sim 1 \mathrm{~T}$, and then increases gradually up to $\sim 6 \mathrm{~T}$. Thus, a substantial portion of quasiparticles is already restored at $\sim 1 \mathrm{~T}$, which is consistent with multiband superconductivity [22]. Above $12 \mathrm{~T}$, $C(H) / T$ increases nearly linearly with $H$ (Fig. 2a). At $\mu_{0} H^{*} \approx 14 \mathrm{~T}, C(H) / T$ exhibits a clear kink and then increases nearly linearly with a smaller slope above $H^{*}$. At $\mu_{0} H \approx 16.4 \mathrm{~T}, C(H) / T$ again exhibits a distinct kink and becomes $H$-independent at higher fields. As $C(H) / T$ is $H$-independent in the normal state, the kink at higher field is attributed to the mean field upper critical field $H_{c 2}^{c}$. Thus, the present results provide thermodynamic evidence for the phase transition at $H^{*}$ well below $H_{c 2}^{c}$. As shown by the blue shaded region in Fig. 2a, substantial portion of the electrons remain condensed into superconducting state just above $H^{*}$. We note that recent measurements of nuclear magnetic resonance report the change of the spin-lattice relaxation rate at $H^{*}[23]$.

The $H$ - $T$ phase diagram displayed in Fig. 3 indicates a noticeable high-field superconducting phase. We denote this phase as $B$-phase (blue shaded regime), distinct from low-field BCS phase ( $A$-phase, yellow shaded regime) [7]. We also determined irreversibility field $H_{i r r}$, at which the vortices become depinned, by the resistivity (Fig. 2c) and the magnetic torque (Fig. 2d). The fact that the $H_{i r r}$ locates between $H^{*}$ and $H_{c 2}^{c}$ firmly establishes that high-field $B$-phase is in the superconducting state. As shown in Fig. 2a, the field linear slope of $C(H) / T$ above $H^{*}$ becomes less steep compared to that below $H^{*}$. This demonstrates that the upper critical field is enhanced by the formation of the high-field $B$-phase. The presence of $B$-phase has also been suggested by the cusp anomalies 


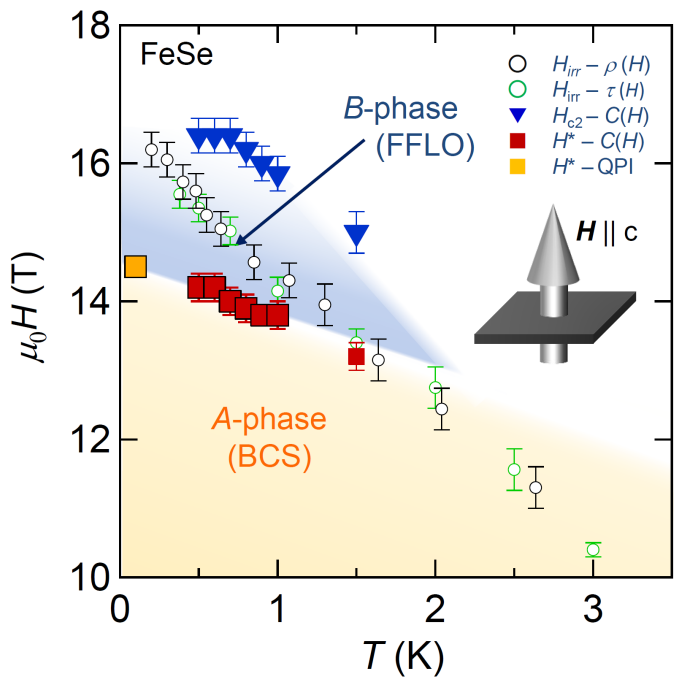

FIG. 3: $H$-T phase diagram of FeSe for $\boldsymbol{H} \| c$ at low temperatures. Blue triangles show the mean-field upper critical field $H_{c 2}^{c}$. Red squares show the kink field $H^{*}$ determined by $C(H) / T$. Orange squre represents $H^{*}$ determined by SI-STM. Black and green circles show $H_{i r r}$ determined by resistivity and magnetic torque, respectively.

of the thermal transport quantities [7, 24]. We stress that the present results provide thermodynamic evidence for the $B$-phase. As the two transition lines at $H^{*}$ and $H_{c 2}^{c}$ are expected to merge at a high temperature, a first order transition occurs at $H^{*}$ or $H_{c 2}^{c}$, or both. However, no discernible hysteresis is observed both at $H^{*}$ and $H_{c 2}^{c}$, implying the weak first order nature of the transition.

To investigate how the superconductivity evolves upon entering the high-field phase, we performed SI-STM at $\sim 90 \mathrm{mK}$ in a wide field range. Figures 4a-d display zero-energy tunnelling conductance $d I(E, \boldsymbol{r}) / d V$ images at various magnetic fields, representing spatial distributions of the local density of states (LDOS) at the Fermi energy. Here, $I(E, \boldsymbol{r})$ is the tunnelling current at energy $E$ and position $\boldsymbol{r}$, and $V$ denotes the sample bias voltage. At $\mu_{0} H=8 \mathrm{~T}$, a vortex lattice is clearly imaged. Each vortex is elliptical due to the nematicity [2527] (Fig. 4a). At $\mu_{0} H=13 \mathrm{~T}$, although the vortexlattice image becomes obscured, the inhomogeneity in the LDOS due to the overlapping of the vortices can be seen (Fig. 4b). At $\mu_{0} H=15 \mathrm{~T}$, the LDOS becomes uniform except for the quasiparticle interference patterns near defects (Fig. 4c), which are also seen in the normal state (Fig. 4d). Fourier-transformed spectroscopic images $d I(E, \boldsymbol{r}) / d V /(I(E, \boldsymbol{r}) / V)$ at $-1.5 \mathrm{meV}$ are shown in Figs. 4e-h. The normal-state quasiparticle interference patterns generate spots along $\boldsymbol{q}_{b}[28]$, whereas vortex and other inhomogeneous LDOS features below $H^{*}$ appear as signals along $\boldsymbol{q}_{a}$. We found that the signals along $\boldsymbol{q}_{a}$ are confined in the energy range below the superconducting gap and are symmetric between filled and empty states (see Fig. S2 and Fig. S3). Therefore, we conclude that the signals along $\boldsymbol{q}_{a}$ are associated with quasiparticles in the superconducting state and their intensity can be used as a measure of superconductivity. To quantify, we subtract the Fourier-transformed $d I(E, \boldsymbol{r}) / d V /(I(E, \boldsymbol{r}) / V)$ in the normal state $(16.5 \mathrm{~T})$ from the ones taken below $H_{c 2}^{c}$. Figures $4 \mathrm{i}-\mathrm{k}$ depict such difference images at $E=-1.5 \mathrm{meV}$. We sum up all the difference images over $|E|< \pm 6 \mathrm{meV}$ and plot its field dependence in Fig. 41 . The superconducting signal is nearly completely suppressed at around $14.5 \mathrm{~T}$, which is plotted by the filled orange square in Fig. 3. As this field lies on an extrapolation of the $H^{*}$ from high temperatures, it is natural to consider that this field corresponds to $H^{*}$.

The most striking feature is that superconducting signal detected by SI-STM vanishes above $H^{*}$ (Fig. 4l), whereas $C(H) / T$ indicates that $\sim 5 \%$ of electrons still remain condensed in the superconducting state at $H^{*}$ (Fig. 2a). These contrasting observations are naturally explained by the formation of the planar node at the top surface of the $a \mathrm{~b}$ plane. This is an expected fingerprint of the FFLO state, in which the planar nodes are formed. This also excludes the possibility that the anomaly of $C(H) / T$ at $H^{*}$ would be caused by the multi-band effect, which should keep the surface superconducting up to $H_{c 2}^{c}$.

There are two possible scenarios of the FFLO state for $\boldsymbol{H} \| c$, in which $\boldsymbol{H}$ is applied parallel to the the quasi$2 \mathrm{D}$ cylindrical Fermi surface. In the first scenario, the Fermi surface is assumed to be purely 2D. In this case, an FFLO state, in which $\boldsymbol{q}$-vector in the $a b$ plane connects the Zeeman split Fermi surface sheets, is stabilized (Fig. 5a). The order parameter oscillates perpendicular to $\boldsymbol{H}$ and periodic nodal planes appear parallel to the vortices (Fig. 5b). This state corresponds to the FFLO state involving Cooper pairs with higher-order Landau levels of index $n \geq 1[3,19-21]$. However, we can exclude this scenario, because superconducting order parameter remains finite in some region of the $a b$ plane, which is inconsistent with the uniform suppression of superconductivity at the top surface. In the second scenario, warping of the cylindrical Fermi surface along the $c$ axis is assumed to be important. In this scenario, FFLO state of the lowest Landau level $(n=0)$, in which $\boldsymbol{q}$ vector parallel to the $c$ axis connects the warped regime of the Zeeman split Fermi surface sheets, is stabilized (Fig. 5c) [21]. The order parameter oscillates along the $c$ axis as $\Delta(z) \propto \sin \left(k_{z} z\right)$ and the periodic planar nodes appear perpendicular to the vortices (Fig. $5 \mathrm{~d}$ ). To place the FFLO nodal planes perpendicular to the $c$ axis, it is natural to consider that one nodal plane is pinned at the top surface of $a b$ plane, which is energetically favoured. This situation can account for the experimental observations.

In FeSe, the pairing interaction is strongly orbital de- 

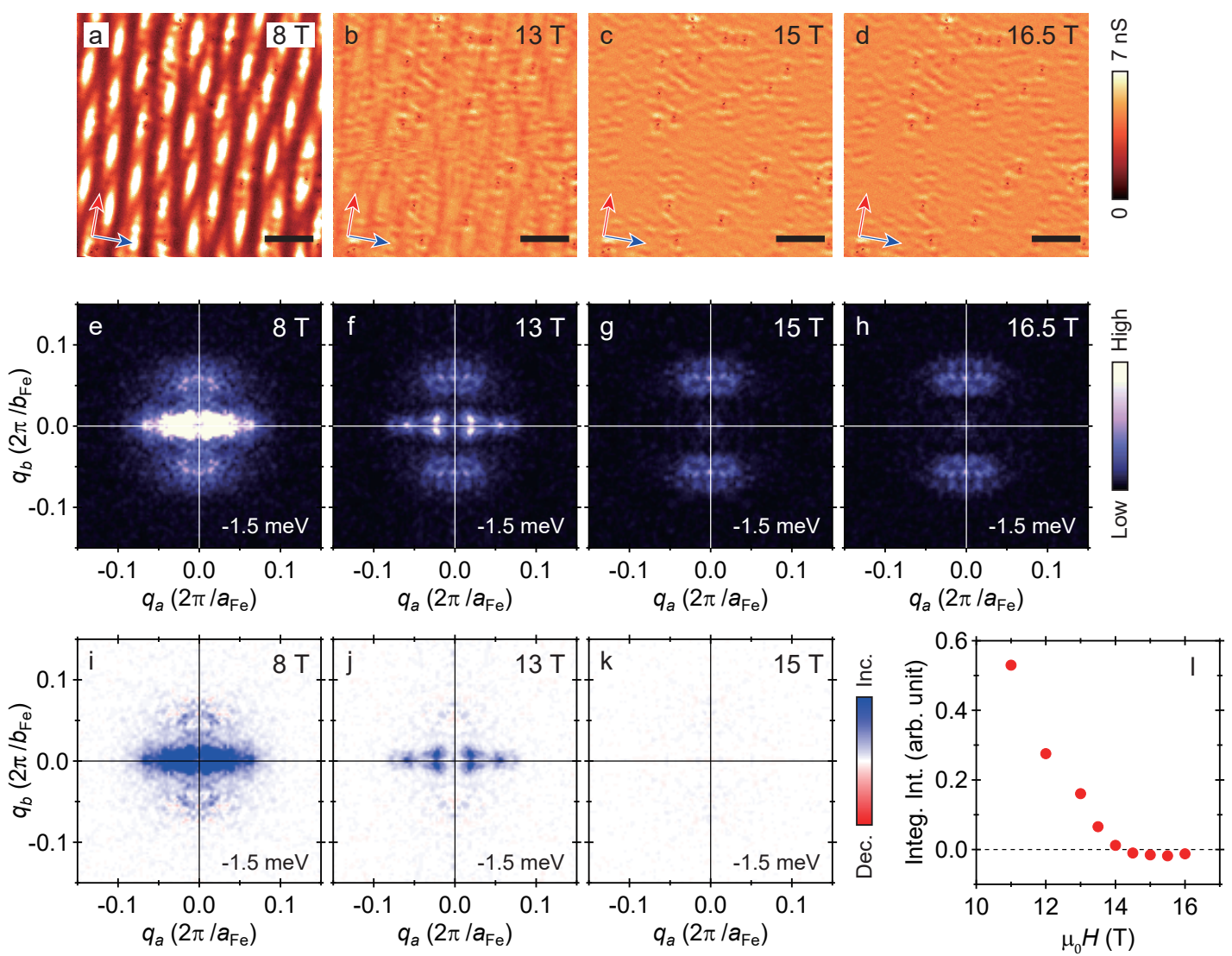

FIG. 4: Spectroscopic images and their Fourier-transformed patterns of FeSe obtained at $T \sim 90 \mathrm{mK}$. a-d, $d I(E=0 \mathrm{meV}, \boldsymbol{r}) / d V$ images at various magnetic fields. Blue and red arrows denote crystallographic $\boldsymbol{a}$ and $\boldsymbol{b}$ directions, respectively. We adopt the coordinate system $|\boldsymbol{a}|<|\boldsymbol{b}|<|\boldsymbol{c}|$. Black scale bars represent $20 \mathrm{~nm}$. At $8 \mathrm{~T}$, each vortex is elongated along the crystallographic $\boldsymbol{b}$ direction. e-h, Fourier-transformed $d I(E, \boldsymbol{r}) / d V /(I(E, \boldsymbol{r}) / V)$ images at $-1.5 \mathrm{meV}$. i-k, Difference images between Fourier-transformed $d I(E, \boldsymbol{r}) / d V /(I(E, \boldsymbol{r}) / V)$ below $H_{c 2}^{c}$ and the one taken at $16.5 \mathrm{~T}>H_{c 2}^{c}$. l, Magnetic field dependence of the integrated intensity of the superconductivity-related signals.

pendent [29]. It has been pointed out that the $d_{x z}$ orbital in the hole pocket plays an important role for the superconductivity, as revealed by the largest superconducting gap in this orbital. Moreover, the $d_{x z}$ orbital has lager weights on the flattened parts of the Fermi surface than the other portion, as illustrated in Figs. 1a and 1b. Therefore it is likely that these parts of Fermi surface with $d_{x z}$ character play an essential role for the FFLO state not only for $\boldsymbol{H} \| a b[16]$ but also $\boldsymbol{H} \| c$. The phase boundary between the BCS and FFLO states is nearly temperature independent for $\boldsymbol{H} \| a b$, whereas it increases as the temperature is lowered for $\boldsymbol{H} \| c$. This may be caused by the orbital pair breaking effect, which is relatively more important for $\boldsymbol{H} \| c$. Emergent highfield phases for both field directions are perhaps the most prominent features in the BCS-BEC crossover superconductor of FeSe, which would provide an unprecedented platform of the long-sought FFLO state.
[1] Fulde, P. \& Ferrell, R. A. Superconductivity in a strong spin-exchange field. Phys. Rev. 135, A550 (1964).

[2] Larkin, A. I. \& Ovchinnikov, Y. N. Inhomogeneous state of superconductors. Sov. Phys. JETP 20, 762 (1965).

[3] Matsuda, Y. \& Shimahara, H. Fulde-Ferrell-LarkinOvchinnikov state in heavy fermion superconductors. $J$. Phys. Soc. Jpn. 76, 051005 (2007).

[4] Wosnitza, J. FFLO States in Layered Organic Superconductors. Ann. Phys. (Berlin) 530, 1700282 (2018).

[5] Kinnunen, J. J., Baarsma, J. E., Martikainen, J.-P. \& Törmä, P. The Fulde-Ferrell-Larkin-Ovchinnikov state for ultracold fermions in lattice and harmonic potentials: a review. Rep. Prog. Phys. 81, 046401 (2018).

[6] Shibauchi, T., Hanaguri, T. \& Matsuda, Y. Exotic Superconducting States in FeSe-based Materials. J. Phys. Soc. Jpn. 89, 102002 (2020).

[7] Kasahara, S. et al. Field-induced superconducting phase of FeSe in the BCS-BEC cross-over. Proc. Natl. Acad. Sci. USA 111, 16309 (2014).

[8] Agosta, C.C. et al. Calorimetric Measurements of Magnetic-Field-Induced Inhomogeneous Superconduc- 


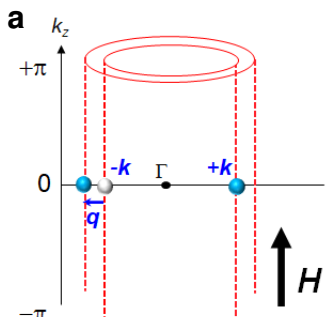

b
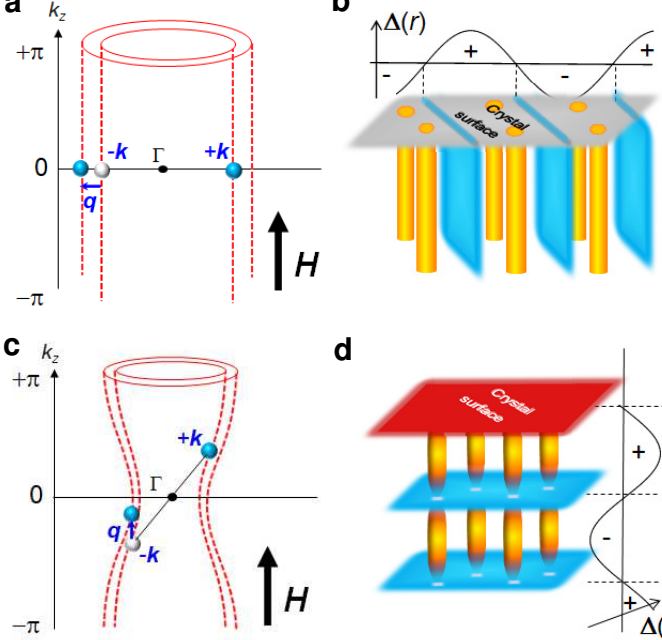

d

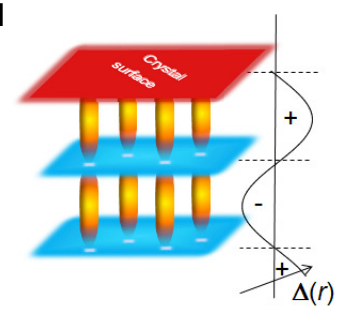

FIG. 5: Schematic illustrations of the possible electron pairing and the nodal structure in the FFLO state. a, Electron pairing with $(\boldsymbol{k} \uparrow,-\boldsymbol{k}+\boldsymbol{q} \downarrow)$ in a purely 2D Fermi surface. For the higher order FFLO state with $n>0$, the $\boldsymbol{q}$ vector orients perpendicular to $\boldsymbol{H}$. b. Schematic illustration of the superconducting order parameter $\Delta$ in real space. c. Electron pairing with $(\boldsymbol{k} \uparrow,-\boldsymbol{k}+\boldsymbol{q} \downarrow)$ in a quasi 2D warped Fermi surface. The $\boldsymbol{q}$ vector orients along the direction of $\boldsymbol{H}$. d, Schematic illustration of the superconducting order parameter $\Delta$ for the $n=0$ FFLO state. The oscillations of the order parameter and the periodic planar nodes appear along the direction of $\boldsymbol{H}$

tivity Above the Paramagnetic Limit Phys. Rev. Lett. 118, 267001 (2017).

[9] Radovan, H. A. et al. Magnetic enhancement of superconductivity from electron spin domains. Nature 425, 51 (2003).

[10] Bianchi, A. et al., Possible Fulde-Ferrell-LarkinOvchinnikov Superconducting State in $\mathrm{CeCoIn}_{5}$. Phys. Rev. Lett. 91, 187004 (2003).

[11] Kreisel, A., Hirschfeld, P. J., \& Andersen, B. M., Symmetry 12, 1402 (2020).

[12] Hsu, F.-C. et al. Superconductivity in the PbO-type structure $\alpha$-FeSe. Proc. Natl. Acad. Sci. USA 105, 1426214264 (2008).

[13] Böhmer, A.E. \& Kreisel, A. Nematicity, magnetism and superconductivity in FeSe J. Phys.: Condens. Matter 30, 023001 (2018).

[14] Coldea, A. I. \& Watson, M. D. The Key Ingredients of the Electronic Structure of FeSe. Annu. Rev. Condens. Matter Phys. 9, 125 (2018).

[15] Yi, M. et al. The Nematic Energy Scale and the Missing Electron Pocket in FeSe. Phys. Rev. X 9, 041049 (2019).

[16] Kasahara, S. et al. Evidence for an Fulde-Ferrell-LarkinOvchinnikov State with Segmented Vortices in the BCSBEC-Crossover Superconductor FeSe. Phys. Rev. Lett. 124, 107001 (2020).

[17] Hardy, F. et al. Vortex-lattice melting and paramagnetic depairing in the nematic superconductor FeSe. Phys. Rev. Research 2, 033319 (2020).

[18] Ok, J. M. et al. Observation of in-plane magnetic field induced phase transitions in FeSe. Phys. Rev. B 101, 224509 (2020).

[19] Shimahara, H. Fulde-Ferrell state in quasi-twodimensional superconductors. Phys. Rev. B 50, 12760 (1994).

[20] Song, K. W. \& Koshelev, A. E. Quantum FFLO State in Clean Layered Superconductors. Phys. Rev. X 9, 021025 (2019).

[21] Shimahara, H. Fulde-Ferrell-Larkin-Ovchinnikov State in Perpendicular Magnetic Fields in Strongly Pauli-Limited Quasi-Two-Dimensional Superconductors. J. Phys. Soc. Jpn. 90, 044706 (2021).

[22] Sato, Y. et al. Proc. Natl. Acad. Sci. USA 115, 1227 (2018).

[23] Molatta, S. et al. Superconductivity of highly spinpolarized electrons in FeSe probed by ${ }^{77} \mathrm{Se}$ NMR. arXiv:2010.10128.

[24] Watashige, T. et al. Quasiparticle Excitations in the Superconducting State of FeSe Probed by Thermal Hall Conductivity in the Vicinity of the BCS-BEC Crossover. J. Phys. Soc. Jpn. 86, 014707 (2017).

[25] Song, C.-L. et al. Direct Observation of Nodes and Twofold Symmetry in FeSe Superconductor. Science 332, 1410 (2011).

[26] Watashige, T. et al. Evidence for time-reversal symmetry breaking of the superconducting state near twinboundary interfaces in FeSe revealed by scanning tunneling spectroscopy. Phys. Rev. X 5, 031022 (2015).

[27] Hanaguri, T. et al. Quantum Vortex Core and Missing Pseudogap in the Multiband BCS-BEC Crossover Superconductor FeSe. Phys. Rev. Lett. 122, 077001 (2019).

[28] Hanaguri, T. et al. Two distinct superconducting pairing states divided by the nematic end point in $\mathrm{FeSe}_{1-x} \mathrm{~S}_{x}$. Sci. Adv. 4, eaar6419 (2018).

[29] Sprau, P. O. et al. Science 357, 75 (2017).

[30] Wang, Y. et al. Direct observation and anisotropy of the contribution of gap nodes in the low-temperature specific heat of $\mathrm{YBa}_{2} \mathrm{Cu}_{3} \mathrm{O}_{7}$. Phys. Rev. B 63, 094508 (2001).

[31] Taylor, O. J., Carrington, A. \& Schlueter, J.A. Specificheat measurements of the gap structure of the organic superconductors $\kappa-(\mathrm{ET})_{2} \mathrm{Cu}\left[\mathrm{N}(\mathrm{CN})_{2}\right] \mathrm{Br}$ and $\kappa$ $(\mathrm{ET})_{2} \mathrm{Cu}(\mathrm{NCS})_{2}$. Phys. Rev. Lett. 99, 057001 (2007).

[32] Machida, T, Kohsaka, Y. \& Hanaguri, T. A scanning tunneling microscope for spectroscopic imaging below 90 $\mathrm{mK}$ in magnetic fields up to $17.5 \mathrm{~T}$. Rev. Sci. Inst. 89, 093707 (2018). 


\section{Methods}

Crystal growth and characterization. Single crystals of FeSe were grown by vapour transport method. Particular attention was paid to select the crystal with extraordinary high-quality. By measuring the STM topography for many crystals, we carefully selected a crystal, in which only 2-3 defects are contained per 10,000 Fe atoms (Fig. S1a) This same single crystal was further cleaved into two pieces. One (smaller) piece $(\sim$ $250 \times 100 \times 5 \mu \mathrm{m}^{3}$ ) was used for the resistivity measurements by directly soldering the four indium contacts. The other (larger) piece $(\sim 30 \mu \mathrm{g})$ was used for the magnetic torque and the heat capacity measurements. In Fig. S1b, we show the temperature dependence of resistivity. The crystal exhibits zero resistivity at $T_{c}=9.0 \mathrm{~K}$. In the inset of Fig. S1b, we plot the temperature dependence of the magnetization $M$ measured by a SQUID magnetometer. A sharp superconducting transition in the $M-T$ curve demonstrates that the sample is homogeneous.

Torque magnetometry. Magnetic torque was measured by the piezo-resistive micro-cantilever technique down to $0.38 \mathrm{~K}$ and up to $\sim 16 \mathrm{~T}$. A tiny single crystal was carefully mounted onto the tip-less piezo-resistive lever (PRS-L450-F30-TL-STD, SCL-Sensor. Tech.) which forms an electrical bridge circuit. The field is slightly tilted away from the $c$ axis. The irreversibility field $H_{i r r}$ is determined by the point at which the hysteresis loop is closed to the level of $0.3 \%$ of the whole signal.

Heat capacity. The heat capacity of the tiny single crystal of FeSe used for the STM and torque measurements was measured by the long-relaxation method $[30,31]$. With a tiny amount of grease, the sample was mounted onto the bare chip Cernox sensor, which is used as a thermometer and a heater. The sensor is suspended from the cold stage by gold-coated glass fibers such that it is weakly linked to the cold stage. The heat capacity of the crystal is obtained by subtracting the addenda from the total heat capacity measured with the sample.

SI-STM. SI-STM experiments were performed with a ultrahigh vacuum dilution-fridge-based STM equipped with a $17.5 \mathrm{~T}$ superconducting magnet [32]. We used a tungsten tip prepared by electrochemical etching. The tip was cleaned by field evaporation using a field-iron microscope, followed by controlled indentation into a clean $\mathrm{Au}(100)$ surface. Clean sample surface was obtained by vacuum cleaving at liquid nitrogen temperature. All data were taken in the constant-current mode with the feedback conditions of $I=100 \mathrm{pA}$ and $V=20 \mathrm{mV}$. $d I / d V$ spectra were taken by standard lock-in technique with a bias modulation of $0.21 \mathrm{mV}_{\text {rms }}$. Whenever we changed the magnetic field, the sample was heated up above $T_{c}$ to ensure uniform vortex distribution in the sample.

\section{Data availability}

The data that support the findings of this study are available on request from the corresponding author.

\section{Acknowledgements}

We thank I. Eremin, R. Ikeda, K. Ishida, S. Kitagawa, H. Kühne, T. Mizushima, S. Molatta, Y. Nagai, J. Wosnitza, and Y. Yanase for stimulating discussion. This work is supported by Grants-in-Aid for Scientific Research (KAKENHI) (Nos. 15H02106, 15H03688, 15KK0160, 18H01177, 18H05227, 19H00649) and on Innovative Areas "Quantum Liquid Crystals" (No. 19H05824) from the Japan Society for the Promotion of Science, and JST CREST (JPMJCR19T5).

\section{Author contributions}

S.K. grew the high-quality single-crystalline samples. S.K. H.S., Y.S. performed resistivity, magnetic torque and heat capacity measurements. T.M. and T.H. performed SI-STM measurements. S.K., H.S., T.M., Y.S., Y.U., H.M., S.S., and T.H. analysed the data. S.K., T.M., Y.K., T.S., T.H. and Y.M. discussed and interpreted the results. S.K., T.S., T.H. and Y.M. prepared the manuscript.

\section{Competing interests}

The authors declare no competing financial interests. 
Figures
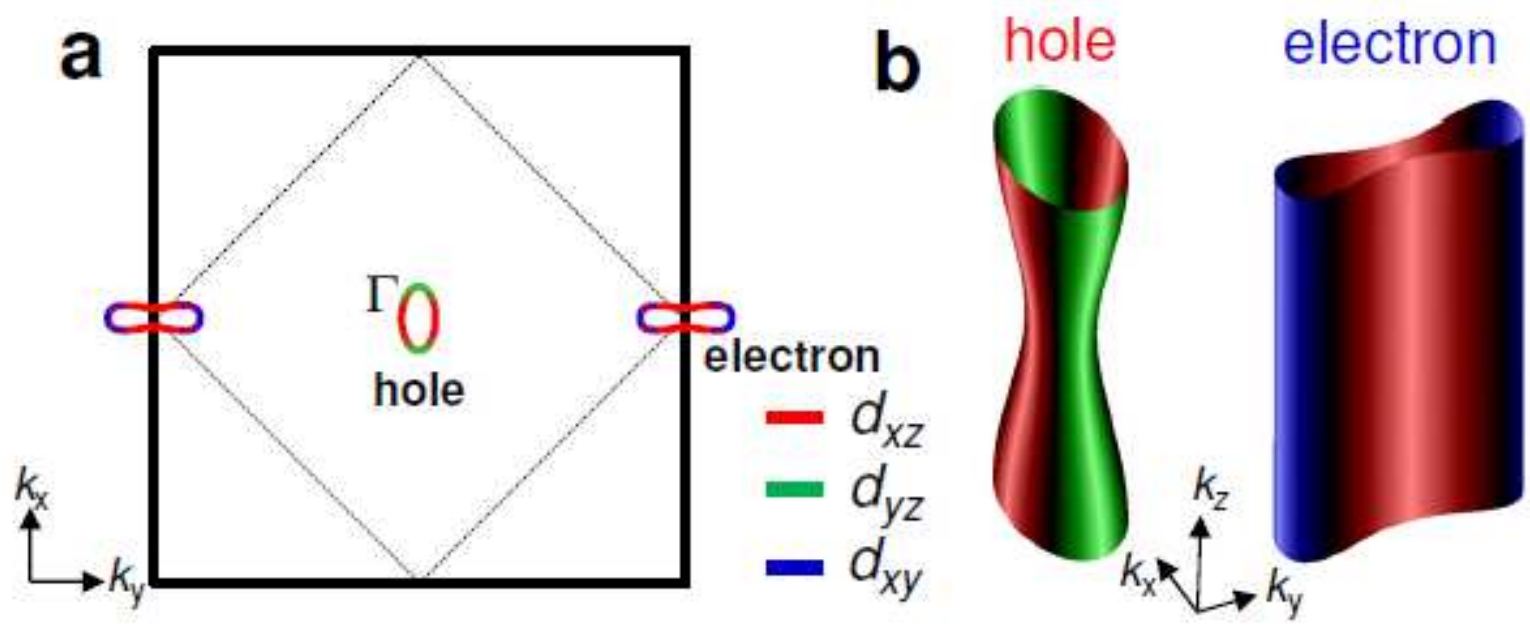

Figure 1

Please see the Manuscript PDF file for the complete figure caption.
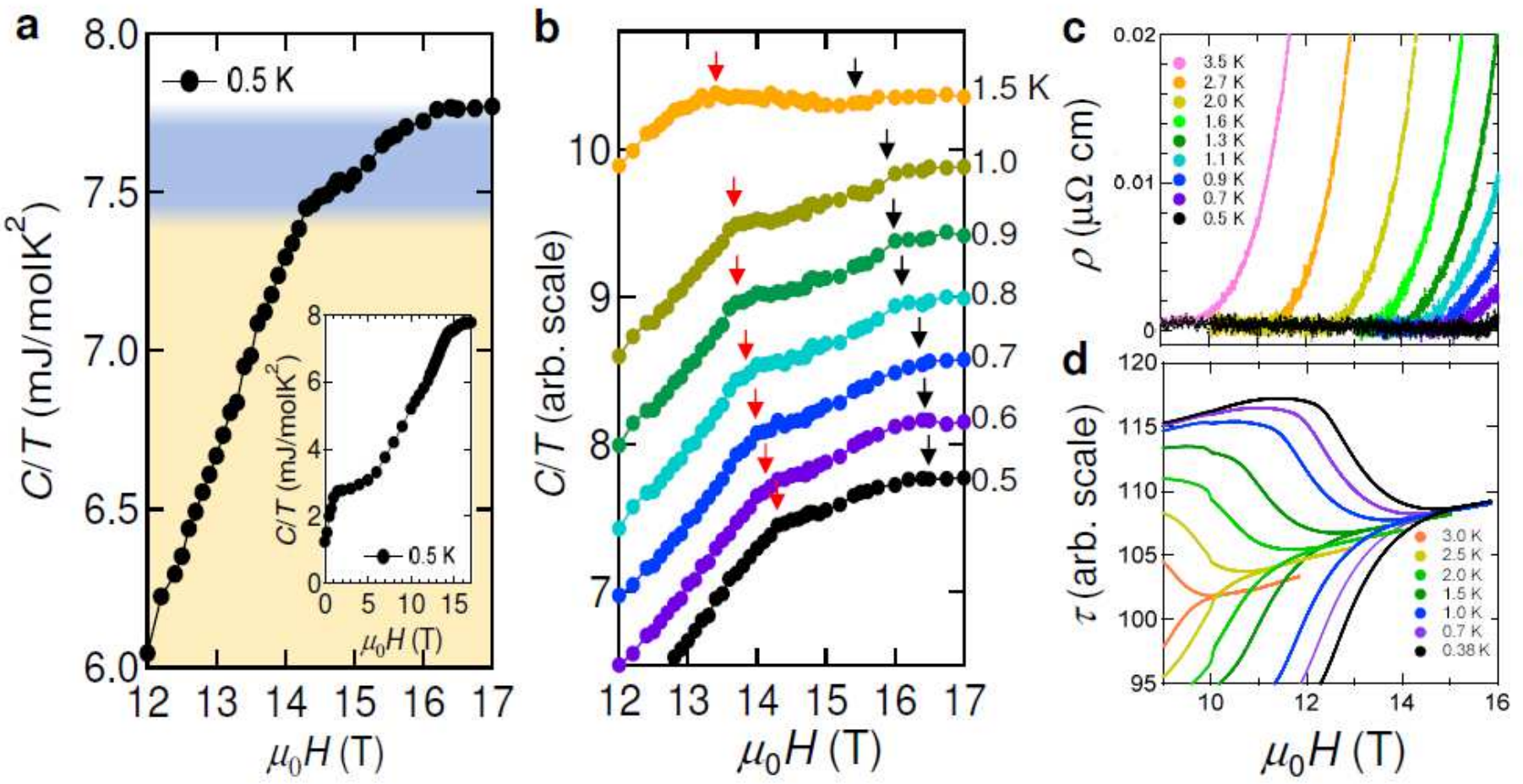

Figure 2

Please see the Manuscript PDF file for the complete figure caption. 


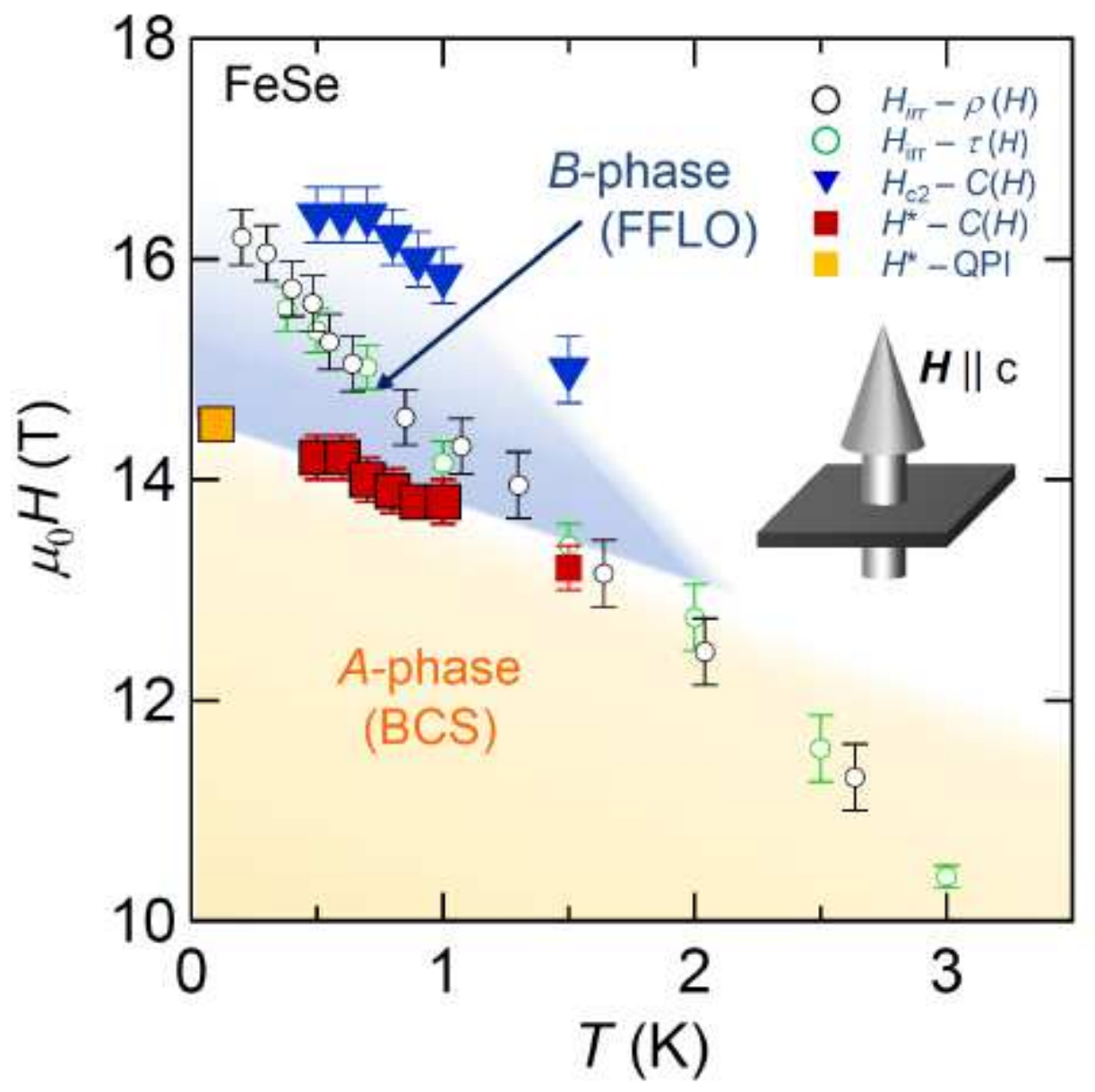

Figure 3

Please see the Manuscript PDF file for the complete figure caption. 

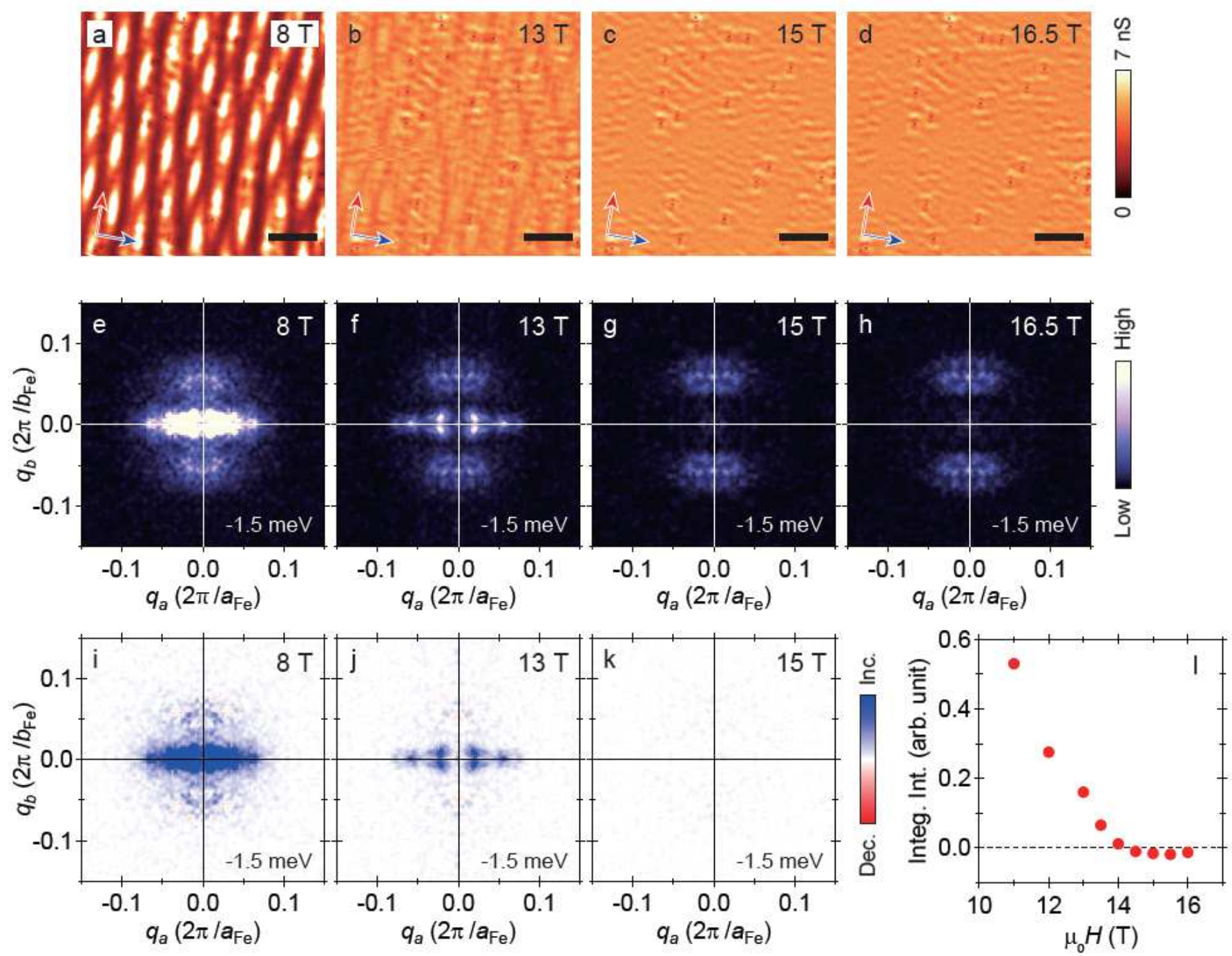

Figure 4

Please see the Manuscript PDF file for the complete figure caption. 


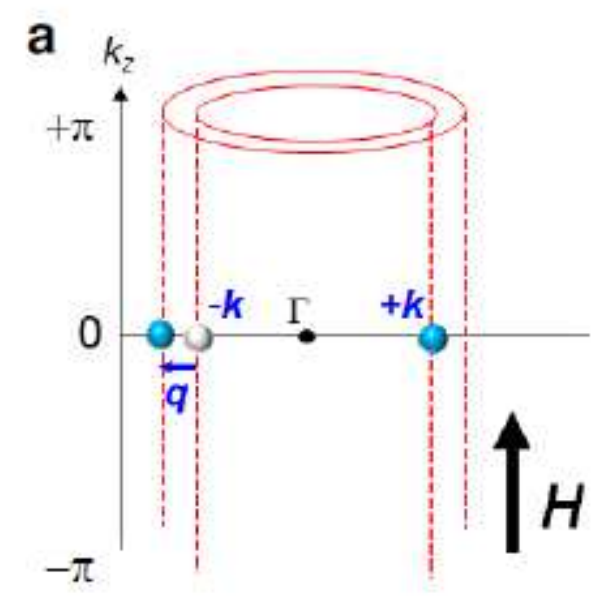

b
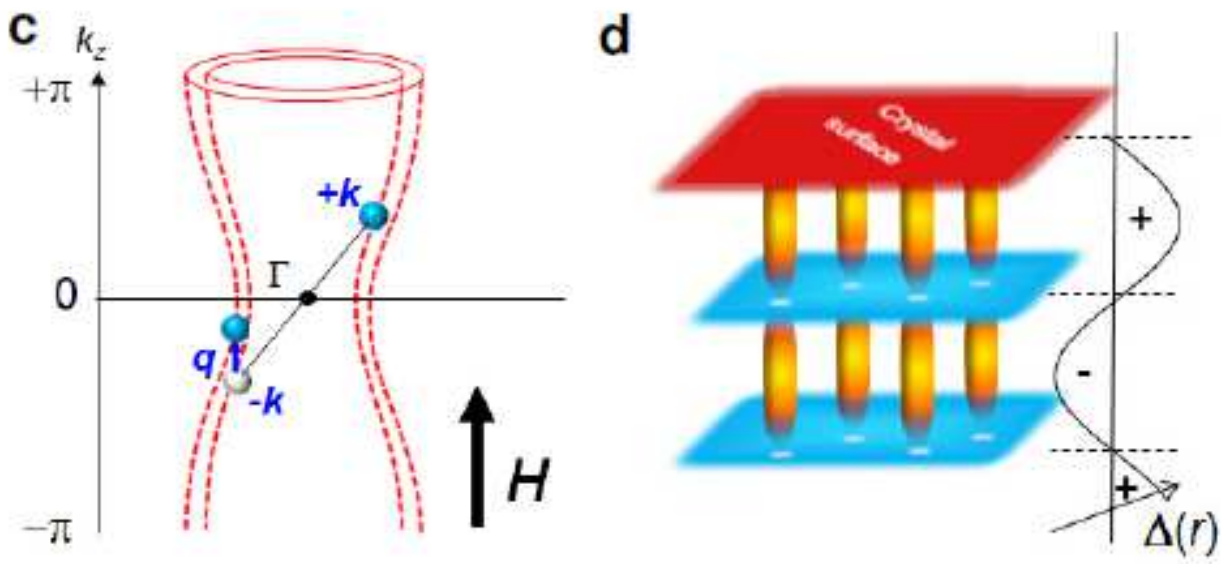

Figure 5

Please see the Manuscript PDF file for the complete figure caption.

\section{Supplementary Files}

This is a list of supplementary files associated with this preprint. Click to download.

- FeSeFFLOcSIF.pdf 